\title{
Long-term epigenetic changes in offspring mice exposed to alcohol during gestation and lactation
}

Lídia Cantacorps $^{1}$, Silvia Alfonso-Loeches ${ }^{3}$, Consuelo Guerri ${ }^{3}$ and Olga Valverde ${ }^{1,2 *}$

${ }^{1}$ Neurobiology of Behaviour Research Group (GReNeC-NeuroBio), Department of Experimental and Health Sciences, Universitat Pompeu Fabra, Barcelona, Spain

${ }^{2}$ Neuroscience Research Programme. IMIM-Hospital del Mar Research Institute, Barcelona, Spain

${ }^{3}$ Molecular and Cellular Pathology of Alcohol, Prince Felipe Research Centre, Valencia, Spain

\section{*Corresponding author:}

Olga Valverde, Neurobiology of Behaviour Research Group (GReNeC-NeuroBio), Department of Experimental and Health Sciences, Universitat Pompeu Fabra. Parc de Recerca Biomèdica de Barcelona. C/ Dr Aiguader 88, 08003 Barcelona, Spain. Email: olga.valverde@upf.edu 


\begin{abstract}
Background:

Alcohol exposure impairs brain development and leads to a range of behavioural and cognitive dysfunctions, termed as foetal alcohol spectrum disorders (FASD). Although different mechanisms have been proposed to participate in FASD, the molecular insights of such effects are still uncertain. Using a mouse model of FASD, we have previously shown that maternal binge-like alcohol drinking causes persistent effects on motor, cognitive and emotional-related behaviours associated with neuroimmune dysfunctions.
\end{abstract}

\title{
Aims:
}

In this study, we sought to evaluate whether the long-term behavioural alterations found in offspring with early exposure to alcohol are associated with epigenetic changes in the hippocampus and prefrontal cortex.

\section{Methods:}

Pregnant C57BL/6 female mice underwent a model procedure for binge alcohol drinking throughout both the gestation and lactation periods. Subsequently, adult offspring were assessed for their cognitive function in a reversal learning task and brain areas were extracted for epigenetic analyses.

\section{Results:}

The results demonstrated that early binge alcohol exposure induces long-term behavioural effects along with alterations in the histone acetylation (H4K5 and H4K12) in the hippocampus and prefrontal cortex. The epigenetic effects were linked with an imbalance in histone acetyltransferase activity that was found to be increased in the prefrontal cortex of mice exposed to alcohol.

\section{Conclusions:}

In conclusion, our results reveal that maternal binge-like alcohol consumption induces persistent epigenetic modifications, effects that might be associated with the long-term cognitive and behavioural impairments observed in FASD models. 


\section{Declaration of Conflicting Interests}

The authors declare that there is no conflict of interest.

\section{Keywords}

Alcohol binge drinking, FASD, cognition, epigenetics, histone acetylation

\section{Abbreviations}

BAC: Blood alcohol concentration

DID: Drinking in the dark

FAS: Foetal Alcohol Syndrome

FASD: Foetal alcohol spectrum disorders

GAPDH: Glyceraldehyde 3-phosphate dehydrogenase

H3K4me3: Histone H3 (tri-methyl lysine 4)

H3K9: Histone H3 lysine 9

H4K5: Histone H4 lysine 5

H4K12: Histone H4 lysine 12

HAT: Histone acetyltransferase

HDAC: Histone deacetylase

HPC: Hippocampus

K-ac: Acetyl Lysine

LMNA: Lamin A/C

PD: Postnatal day

PFC: Prefrontal cortex

PLAE: Prenatal and lactation alcohol exposed 


\section{Introduction}

Alcohol is one of the most commonly consumed psychoactive substances worldwide with dependence-producing properties. Although it is well known that the consumption of alcohol during foetal development has teratogenic effects, it is still one of the main preventable causes of birth defects and intellectual and neurodevelopmental disabilities (May et al., 2018). The set of physical and cognitive defects observed in children born of mothers who drank during pregnancy are grouped under the umbrella term Foetal Alcohol Spectrum Disorders (FASD) (Dörrie et al., 2014; Guerri et al., 2009; Hoyme et al., 2016). Despite its preventability, the global prevalence of FASD among children and young adults was estimated to be 7.7 per 1000 (Lange et al., 2017). The resulting neurobehavioural outcome of prenatal alcohol exposure may depend on the developmental timing of alcohol exposure and the dose and pattern of exposure, among other factors (Alfonso-Loeches and Guerri, 2011; Kleiber et al., 2014; Sadrian et al., 2014). Therefore, a binge-like exposure may have a greater impact on brain development than a moderate exposure, producing less severe defects (Barron et al., 2016).

In recent years, binge drinking has become a popular pattern of alcohol consumption among teenagers and young adult populations in western countries, and also in pregnant women, which is a cause of great concern. Indeed, it has been reported that approximately $3 \%$ of women engaged in binge drinking during pregnancy (Popova et al., 2017). However, until now, scarce preclinical studies have evaluated the long-term consequences induced by maternal alcohol binge drinking during gestation and lactation. In previous studies we have shown that maternal binge-like alcohol consumption induces persistent behavioural and molecular alterations in the brain of adult offspring mice (Cantacorps et al., 2017; 2018), such as neuroinflammation and myelin damage, specifically in the prefrontal cortex (PFC) and the hippocampus (HPC), which are involved in cognitive processes and executive functions (Lara and Wallis, 2015; Rubin et al., 2014; Sigurdsson and Duvarci, 2016), and that these effects might be underlying some of the cognitive and behavioural impairments observed in adulthood. Alterations in hippocampal neurogenesis and functional brain networks connectivity were also reported in our mouse model (Cantacorps et al., 2018), underlying some of 
the mechanistic bases of FASD etiology. Moreover, the role of epigenetic changes in alcohol-induced damage has been highlighted in recent years (Liyanage et al., 2017; Mahnke et al., 2017; Shukla et al., 2008). It has been shown that alcohol is capable of modifying epigenetics, triggering alterations in gene expression. Alcohol and/or its metabolites, acetaldehyde and acetate may induce alterations in histone acetylation and methylation by altering the activity of the enzymes that mediate these processes, such as histone deacetylases (HDACs) and histone acetyltransferases (HATs) (Zakhari, 2013). For instance, it has been shown that alcohol produces the acetylation of histone $\mathrm{H} 3$ at lysine 9 (H3K9), which is related to gene upregulation (Krishnan et al., 2014). Furthermore, chronic alcohol abuse in humans may result in global increases in trimethylation levels of histone 3 at the lysine $4(\mathrm{H} 3 \mathrm{~K} 4 \mathrm{me} 3)$, which is a promoterenriched chromatin mark of actively transcribed genes (Ponomarev, 2013). In addition, increased levels of histone 4 acetylation at the lysine 5 (H4K5ac) and lysine 12 (H4K12ac) have been reported in the PFC of adolescent mice after binge alcohol drinking (Montesinos et al., 2016), and in the PFC and in the nucleus accumbens after an intermittent alcohol treatment (Pascual et al., 2009).

Gestation and lactation periods are significantly vulnerable to epigenetic alterations, as active changes occur in the epigenome as part of developmental processes (Banik et al., 2017). Some reports have highlighted epigenetic imbalance in foetuses due to developmental alcohol exposure (Basavarajappa and Subbanna, 2016; Liyanage et al., 2017; Ramsay, 2010). Epigenetic alterations induced by in utero alcohol exposure include post-translational modifications of histones, such as acetylation and methylation, which may give rise to changes in the expression of several genes during development (Mandal et al., 2017). Indeed, early alcohol exposure has been shown to alter the epigenetic regulation of genes involved in imprinting, neural and glial development, cell cycle regulation and nervous system growth (Krishnan et al., 2014; Resendiz et al., 2014). Using a second-trimester binge alcohol exposure model in mice, it has been shown that alcohol induced expression changes in genes associated with apoptosis, cellular redox and inflammation as a short-term effect, while differential expression in brain miRNAs was maintained into adulthood (Mantha et al., 2014). Furthermore, alterations in DNA methylation profiles have been found in alcoholexposed rodent offspring, associated with changes in the expression of several genes 
involved in multiple biological functions (Liu et al., 2009; Marjonen et al., 2015; Ungerer et al., 2013).

In this context, the present study addresses the possible association between epigenetic alterations induced by maternal binge-like alcohol drinking and the neurobehavioural outcome in offspring mice using the drinking in the dark (DID) test (Rhodes et al., 2005), a model of voluntary alcohol consumption with time-limited access, which mimics binge drinking during pregnancy and breastfeeding. In particular, posttranslational histone modifications as well as the activity of the HAT and HDAC enzymes in the PFC and the HPC were evaluated in prenatal and lactational alcohol exposed (PLAE) adult offspring mice. Epigenetic marks assessed herein play an important role in learning and memory gene expression, which strengths the importance of transcriptional regulation programs in cognitive processes. For instance, acetylation of H4K5 and H4K12 have been related with hippocampal memory in mice (Park et al., 2013; Peleg et al., 2010; Sweatt, 2010). Therefore, in this study we attempt to provide evidence of the link between cognitive dysfunction and epigenetic changes in offspring mice exposed to alcohol during key periods for brain development. 


\section{Materials and methods}

\section{Animals}

In the present study, data is obtained from the same cohort of animals used in our previous report (Cantacorps et al., 2017), while we tested a separate group of animals from the same cohort of breeding pairs used in this previous study for the behavioural assessment in the T-maze. Twelve week old male and female C57BL/6 inbred mice $(\mathrm{n}=28)$ were purchased from Charles River (Barcelona, Spain) and shipped to our animal facility (UBIOMEX, PRBB). Upon arrival, they were all housed in standard cages at a constant temperature $\left(21 \pm 1^{\circ} \mathrm{C}\right)$ and humidity $(55 \pm 10 \%)$, with a reverse light-dark cycle (white lights on 20:00-08:00 h). After one week of acclimatization, breeding pairs were mated and pregnant females were observed daily for parturition. For each litter, the date of birth was designated as postnatal day (PD) 0. Pups remained with their mothers for 21 days and were subsequently weaned (PD21). After weaning, male offspring were housed in groups of 4. Female offspring were used for other experiments. Food and water were available ad libitum except during the DID procedure, during which the original water bottles were replaced by small bottles containing either water or alcohol solution and food was removed, as below mentioned. Every effort was made to minimize the number of animals used and their suffering. All animal care and experimental procedures were conducted in accordance with the European Communities Directive 86/609/EEC regulating animal research and were approved by the local ethics committee (CEEA-PRBB).

\section{Drugs}

Ethyl alcohol was purchased from Merck Chemicals (Darmstadt, Germany) and diluted in tap water in order to obtain a $20 \%(\mathrm{v} / \mathrm{v})$ alcohol solution.

\section{Drinking-in-the-dark test (DID test)}

This procedure was conducted as previously reported (Cantacorps et al., 2017), commencing two days after mating. Pregnant females were randomly assigned to two groups: alcohol and water-exposed (control) ( $\mathrm{n}=14$ per group). Briefly, the food was removed and the water bottles were replaced with $10-\mathrm{ml}$ graduated cylinders fitted with sipper tubes containing either $20 \%$ (v/v) alcohol in tap water or only tap water (control group) $3 \mathrm{~h}$ after the lights were turned off. Following a $2 \mathrm{~h}$-access period, individual 
intake was recorded and food and the original water bottles were returned to the home cage. During this time, female mice were individually housed and each corresponding male breeding pair was removed from the home cage for the DID procedure. The procedure was repeated on days 2 and 3 and fresh fluids were provided each day (from Monday to Wednesday). On day 4 (Thursday), alcohol or water cylinders were left for $4 \mathrm{~h}$ instead of $2 \mathrm{~h}$, corresponding to the binge drinking session, and fluid intakes were recorded. Two empty control cages (water and alcohol) were placed in the rack to measure general liquid loss (leakage/evaporation) and drip values were subtracted from the drinking values. Fluid intakes (g/kg body weight) were calculated on the basis of average 2-day body weight values, as dams were weighed at 2-day intervals (Mondays and Wednesdays). The procedure was maintained throughout the 3-week gestation and

the 3-week lactation periods. As previously reported (Cantacorps et al., 2017), blood alcohol concentration (BAC) for dams reached levels of $\sim 80 \mathrm{mg} / \mathrm{dl}$ following the last binge drinking session (4h-access) of gestation and lactation periods. A diagram of general experimental procedure is presented in Figure 1.

\section{Maternal care behaviour}

Spontaneous maternal behaviour was recorded 3 times per day (08:00, 13:00 and 17:00) from PD1 to PD7 as previously reported, with minor modifications (Fodor et al., 2012; Gracia-Rubio et al., 2016). In short, the behaviour of each mother ( $n=6$ per group) was scored 20 times at 3 -minute intervals (20 observations x 3 periods per day $\times 7$ days $=$ 420 observations/mother). The following behaviours were scored as present or absent and quantified in a checklist: 1) mother licking and grooming offspring; 2) mother nursing offspring in an "arched-back" posture with rigid limbs; 3) mother nursing in a "blanket" posture; 4) mother nursing in a "passive" posture; 5) mother "out of the nest" (no maternal contact); and 6) "nest-building".

\section{Neonatal offspring assessment}

Litters were left undisturbed until PD5 at which time litter size was determined. Weights of the entire litter were collected on PD5 and 21 and average pup weight was determined by dividing the total litter weight by the number of pups present in each litter as previously described (Brady et al., 2012).

\section{Left-right discrimination learning}


Adult offspring male mice (PD60; n=10 per group) were assessed for left-right discrimination learning using a T-maze (length of stem $30 \mathrm{~cm}$, length of arms $30 \mathrm{~cm}$, width $12 \mathrm{~cm}$, wall height $16 \mathrm{~cm})$ filled with water $\left(23 \pm 2{ }^{\circ} \mathrm{C}\right)$ as previously described (Filali and Lalonde, 2009). A platform $(11$ x $11 \mathrm{~cm})$ was submerged at the end of each arm $1 \mathrm{~cm}$ beneath the surface. Turning preference was tested during the first two trials. The platform was then placed on the least preferred arm, counterbalancing the number of mice reinforced on either side. The mice were placed in the stem of the T-maze and swam either to the left or to the right until finding the submerged platform for a maximum of $60 \mathrm{~s}$. After reaching the platform, mice were removed from the maze to allow them to recover and then placed back in the maze for up to a maximum of 25 trials. Acquisition criterion was considered to be achieved when a mouse reached the platform in 5 consecutive trials. Once the acquisition criterion was acquired, animals did not perform any other trial. After $48 \mathrm{~h}$, the reversal learning phase was conducted, in which the escape platform was placed on the opposite arm. Latency to reach the platform and number of trials to reach criterion were recorded.

\section{Sample preparation and western blot analysis}

Total protein extracts were isolated from the PFC and HPC areas $(250 \mathrm{mg}$ tissue $/ 0.5 \mathrm{ml}$ lysis buffer) taken from adult mouse brains (PD70; $n=3-5$ per group). The tissue was first homogenized in cold lysis buffer (1\% Nonidet P-40, $20 \mathrm{mM}$ Tris- $\mathrm{HCl}$ pH8, 130 $\mathrm{mM} \mathrm{NaCl}, 10 \mathrm{mM} \mathrm{NaF}, 10 \mu \mathrm{g} / \mathrm{ml}$ aprotinin, $10 \mu \mathrm{g} / \mathrm{ml}$ leupeptin, $10 \mathrm{mM}$ DTT, $1 \mathrm{mM}$ Na3VO4 and $1 \mathrm{mM}$ PMSF). Homogenates were kept on ice for $30 \mathrm{~min}$, centrifuged at maximum speed for 15 min., and the supernatant was collected to determine protein concentration. Also, to determine nuclear proteins, we isolated the cytoplasmic and nuclear extracts using the Epiquik Nuclear Extraction kit and carefully following the commercial guidelines (Epigentek, Farmingdale, NY). The lysate protein concentration was measured using the Pierce BCA (bicinchoninic acid) assay kit (Thermo Fisher Scientific, Spain). Lysates were then separated by SDS-PAGE gels and immunoblotting was performed as previously described (Alfonso-Loeches et al., 2010). Membranes were incubated overnight at $4^{\circ} \mathrm{C}$ with primary antibodies (see Table 1, below). After washing with TBS/T, blots were incubated with their respective HRP-conjugated secondary antibodies: anti-mouse (1/5000, Sigma Aldrich) or anti-rabbit (1/20000, Sigma Aldrich). Blots were developed using the ECL system (ECL Plus, Amersham Biosciences). Some membranes were stripped for $1 \mathrm{~h}$ at $60^{\circ} \mathrm{C}$ in SDS solution $(2 \%$ 
SDS, 0.85\% 2-ME, and $65 \mathrm{mM}$ Tris-HCl (pH 6.8), washed, and incubated with antiGAPDH (1/3000, Chemicon) or anti-Lamin A/C (LMNA; 1/1000, Cell Signalling) for 2 $\mathrm{h}$ as loading controls. A densitometry analysis was assessed with Alpha-Ease FC, version Alpha Imager 2200 (Alpha Innotech Corporation). For comparative purposes, control values were normalized to $100 \%$ and the respective protein expression values were adjusted according to the normalization factor.

Table 1. Primary antibodies.

\begin{tabular}{|ccccc|}
\hline Antibody & Description & Host & Dilution & Company \\
\hline H3K9ac & Histone H3 Acetyl-K9 & Rabbit & $1: 1000$ & Cell signalling \\
\hline H4K5ac & Histone H4 Acetyl-K5 & Rabbit & $1: 10000$ & Abcam \\
\hline H4K12ac & Histone H4 Acetyl-K12 & Rabbit & $1: 1000$ & Cell signalling \\
\hline K-Ac & Acetyl Lysine & Rabbit & $1: 1000$ & Abcam \\
\hline H3K4me3 & Histone H3 (tri-methyl k4) & Rabbit & $1: 1000$ & Abcam \\
\hline LMNA & Lamin A/C & Mouse & $1: 1000$ & Cell Signalling \\
\hline GAPDH & Glyceraldehyde 3-phosphate dehydrogenase (also & Mouse & $1: 3000$ & Chemicon \\
& known as G3PDH) & & & \\
\hline
\end{tabular}

\section{Measurement of activity of histone acetyltransferase and histone deacetylase}

The activity of HAT and HDAC was measured in the nuclear fraction using the EpiQuik $^{\text {TM }}$ HAT Activity/Inhibitor Assay Kit (Epigentek, Madrid, Spain), and the Epigenase HDAC Activity/Inhibition Direct Assay Kit (Epigentek, Madrid, Spain), respectively. In these kits, the microplates contain the respective substrates stably coated onto the wells. The nuclear extract samples of PFC and HPC were added to catalyse the intended reaction. The products were then detected in ELISA-like reactions using specific antibodies, as indicated in the manufacturer's instructions. Each sample's optical density (OD) was measured at $450 \mathrm{~nm}$ and the results were expressed as $\mathrm{OD} / \mathrm{h} / \mathrm{mg}$ protein.

\section{Statistical analysis}

One-way analysis of variance (ANOVA) with repeated measures was used to analyse maternal alcohol intake during the DID test. Maternal care data were analysed using 
two-way ANOVA with repeated measures. Subsequent Bonferroni post-hoc comparisons were calculated when required. Behavioural and biochemical data were analysed using unpaired two-tailed Student's t-test. Correlations between maternal alcohol intake levels and behavioural and neurochemical results were performed by Pearson's correlation analyses. In all experiments, results are given as [mean $\pm \mathrm{SEM}$ ], and differences were considered significant when the probability of error was less than 5\%. All statistical analyses were performed using SPSS software (version 23.0). 


\section{Results}

\section{Maternal alcohol intake during prenatal and postnatal DID procedure}

The alcohol intake during binge drinking sessions, corresponding to the $4 \mathrm{~h}$-access day of each week, is shown in Figure 2. The means \pm SEM of the weekly binge episodes during gestation and lactation periods were: $8.14 \pm 0.572 \mathrm{gEtOH} / \mathrm{kg}$ (first week); $5.94 \pm$ $0.544 \mathrm{gEtOH} / \mathrm{kg}$ (second week); $6.38 \pm 0.519 \mathrm{gEtOH} / \mathrm{kg}$ (third week); $5.5 \pm 0.493$ $\mathrm{gEtOH} / \mathrm{kg}$ (fourth week); $5 \pm 0.69 \mathrm{gEtOH} / \mathrm{kg}$ (fifth week) and $7.26 \pm 0.306 \mathrm{gEtOH} / \mathrm{kg}$ (sixth week), respectively. One-way ANOVA with repeated measures showed no effect of day $[\mathrm{F}(13,65)=0.914 ; \mathrm{p}=\mathrm{n} . \mathrm{s}$.]. All subjects could be considered high consumers according to our criteria, since individual alcohol intake during binge sessions was higher than $5 \mathrm{gEtOH} / \mathrm{kg}$.

\section{Maternal care behaviour is not affected by binge-like alcohol drinking}

To evaluate the effect of maternal alcohol consumption during gestation and lactation periods on maternal behaviour, spontaneous maternal care was observed from PD1 to 7 (Fig. 3). Two-way ANOVA of arched-back nursing, blanket posture, passive posture and off-nest behaviour showed an effect of day in all the assessed postures and no effect of group in any posture, but a significant interaction between these factors was observed in terms of arched-back nursing and off-nest behaviour (Table 2).

Bonferroni post-hoc comparisons showed significant differences between water- and alcohol-exposed dams in arched-back nursing on days 4 and $6(\mathrm{p}<0.05)$, and in off-nest behaviour on day $4(\mathrm{p}<0.05)$.

Table 2. Two-way ANOVA calculated for maternal care behaviour of dams exposed to DID test during prenatal and postnatal periods.

\begin{tabular}{|llllllllll|}
\hline & A) Arched-back & B) Blanket & & C) Passive & & D) Out of nest \\
& $F$ & $p<$ & $F$ & $p<$ & $F$ & $p<$ & $F$ & $p<$ \\
\hline Day & $\mathrm{F}(6,60)=11.830$ & 0.001 & $\mathrm{~F}(6,60)=6.746$ & 0.001 & $\mathrm{~F}(6,60)=3.372$ & 0.01 & $\mathrm{~F}(6,60)=6.67$ & 0.001 \\
\hline Group & $\mathrm{F}(1,10)=4.602$ & n.s. & $\mathrm{F}(1,10)=1.331$ & n.s. & $\mathrm{F}(1,10)=0.615$ & n.s. & $\mathrm{F}(1,25)=2.773$ & n.s. \\
\hline Interaction & $\mathrm{F}(6,60)=3.247$ & 0.01 & $\mathrm{~F}(6,60)=1.935$ & n.s. & $\mathrm{F}(6,60)=0.806$ & n.s. & $\mathrm{F}(6,60)=2.474$ & 0.05 \\
\hline
\end{tabular}




\section{Litter size and offspring weight is not affected by maternal alcohol consumption}

Litter size was determined at PD5. The average litter size between groups was not significantly different $\left(\mathrm{t}_{21}=0.047\right.$, n.s.; Table 3). Offspring weight was also measured at PDs 5 and 21 (Table 3). Two-way ANOVA analysis showed a significant effect of time point $[\mathrm{F}(1,21)=1343 ; \mathrm{p}<0.001]$ but no effect of group $[\mathrm{F}(1,21)=0.139$; n.s. $]$ and no interaction $[\mathrm{F}(1,21)=0.415$; n.s. $]$ between factors.

Table 3. Litter size and average pup weight. Values are [mean \pm SEM].

\begin{tabular}{|lll|}
\hline & Control $(\mathbf{n}=\mathbf{1 0})$ & PLAE $(\mathbf{n = 1 3})$ \\
\hline Litter size (pups per litter) & $7.9 \pm 0.4$ & $7.9 \pm 0.3$ \\
\hline Pup weight at PD5 (g) & $2.9 \pm 0.1$ & $2.7 \pm 0.1$ \\
\hline Pup weight at PD21 (g) & $8.2 \pm 0.2$ & $8.2 \pm 0.3$ \\
\hline
\end{tabular}

\section{Prenatal and lactational alcohol exposure induces mild learning acquisition deficits}

\section{later in adulthood}

PLAE and control mice were assessed for left-right discrimination learning in a waterfilled T-maze. Analysis of escape latencies during the last 5 trials prior to reaching criteria during acquisition and reversal learning phases showed a significant effect of trials $[\mathrm{F}(9,171)=4.495 ; \mathrm{p}<0.001]$, with no group effect $[\mathrm{F}(1,19)=0.425 ;$ n.s. $]$ and no interaction between factors $[F(9,171)=0.764$; n.s.] (Fig. 4A). Regarding the number of trials needed to reach acquisition criterion, the unpaired Student's t-test showed a significant difference between alcohol- and water-exposed mice $\left(\mathrm{t}_{20}=2.13 ; \mathrm{p}<0.05\right)$. In contrast, during reversal learning phase, no significant differences were found between groups $\left(\mathrm{t}_{20}=1.29 ; \mathrm{p}=\mathrm{n} . \mathrm{s}\right.$.) (Fig. $\left.4 \mathrm{~B}\right)$.

In addition, correlation between the levels of maternal alcohol intake and the number of trials needed to reach criterion during acquisition phase was assessed by Pearson's correlation analysis, but no significant correlation was found $(r=-0.44 ; p=n . s$.$) .$

Maternal binge-like alcohol consumption induces long-lasting epigenetic modifications in offspring 
We used western blot to analyse the levels of histone acetylation and methylation and to ascertain whether alcohol exposure in the developing brain triggers long-term epigenetic changes.

Table 4. Unpaired Student's t-test for histone modification measurements in the PFC and the HPC of PLAE mice compared with the control group.

\begin{tabular}{|lllll|}
\hline & A) PFC & & B) HPC & \\
\hline & $t$-ratio & $p<$ & $t$-ratio & $p<$ \\
\hline H3K9 & $\mathrm{t}_{8}=1.550$ & n.s. & $\mathrm{t}_{8}=0.337$ & n.s. \\
\hline H4K5 & $\mathrm{t}_{8}=2.423$ & 0.05 & $\mathrm{t}_{8}=4.050$ & 0.05 \\
\hline H4K12 & $\mathrm{t}_{8}=3.744$ & 0.05 & $\mathrm{t}_{8}=2.278$ & n.s. \\
\hline K-ac & $\mathrm{t}_{8}=2.743$ & 0.05 & $\mathrm{t}_{8}=0.549$ & n.s. \\
\hline H3K4me3 & $\mathrm{t}_{6}=1.350$ & n.s. & $\mathrm{t}_{6}=1.332$ & n.s. \\
\hline
\end{tabular}

Student's t-test revealed significant differences in acetylation levels of H4K5, H4K12 and total K-ac in the PFC of PLAE offspring mice (Fig. 5 and Table 4). However, no significant alterations were found in $\mathrm{H} 3 \mathrm{~K} 9$ acetylation and $\mathrm{H} 3 \mathrm{~K} 4 \mathrm{me} 3$ levels. In the HPC (Fig. 5) we found significantly increased H4K5 acetylation levels (Table 4), while the other parameters showed no significant differences when compared with the control group.

Furthermore, Pearson's correlation analyses between epigenetic modifications and maternal alcohol consumption showed a significant correlation between the H4K5ac levels $(r=0.87 ; \mathrm{p}<0.05)$ in the PFC and the maternal alcohol intake. None of the other correlation analyses were statistically significant.

\section{Changes in histone acetyltransferase and histone deacetylase in adult offspring exposed to alcohol during gestation and lactation}

We also assessed the activity of enzymes that regulate the acetylation and deacetylation of histones, the HAT and the HDAC, in different brain areas of adult offspring mice.

Statistical analyses showed that prenatal and early postnatal alcohol exposure significantly increased the HAT activity in the PFC $\left(\mathrm{t}_{6}=2.747 ; \mathrm{p}<0.05\right)$, although it remained unchanged in the HPC $\left(\mathrm{t}_{6}=1.238\right.$; n.s.) (Fig. 6A). Moreover, a non-significant 
tendency was observed towards reduced HDAC activity in both the PFC $\left(\mathrm{t}_{6}=2.226\right.$; $\mathrm{p}=0.06)$ and the HPC $\left(\mathrm{t}_{6}=2.120 ; \mathrm{p}=0.07\right)$ of PLAE mice (Fig. $\left.6 \mathrm{~B}\right)$. 


\section{Discussion}

Alcohol is one of the most significant neurotoxic compounds to affect the developing brain. Exposure to alcohol during foetal development can impair brain ontogeny, leading to significant long-lasting neurocognitive and behavioural dysfunctions (Drew and Kane, 2014; Murray et al., 2016; Zuccolo et al., 2013). Epigenetic factors (ie. DNA methylation, post-translational histone modifications and small non-coding RNAs) during brain ontogeny are emerging as key regulators of neural plasticity, producing changes in dendritic branching and spine density, with subsequent enduring changes of neuronal functioning and behaviour (Keverne et al., 2015; Kundakovic and Champagne, 2015; Petanjek and Kostovic, 2012).

Using a FASD model of binge-like alcohol exposure in mice, we provide evidence that alcohol-induced long-term behavioural effects are associated with epigenetic changes, such as alterations of histone acetylation and methylation levels together with alterations in HAT and HDAC enzyme activities in two brain structures particularly affected by alcohol exposure, the PFC and the HPC. We have also shown that maternal alcohol consumption during early-life periods causes long-lasting effects on motor coordination, cognitive and emotional-related behaviours associated with long-term neuroimmune dysfunctions, myelin disruptions and alterations in functional brain connectivity (Cantacorps et al., 2017; 2018).

In accordance with our results, other studies have demonstrated that alcohol exposure in utero may have an impact on epigenetic processes (Chater-Diehl et al., 2017; Lussier et al., 2017) inducing long-term genomic and epigenomic dysregulations that may account for FASD etiology (Haycock, 2009; Kleiber et al., 2014; Resendiz et al., 2013). Moreover, the early-life period is a time of particular susceptibility to epigenetic alterations, which can be modified by environmental factors, such as maternal care. The mother-pup interaction plays an important role during the first postnatal days of life, as variations in maternal behaviour can induce differences in the stress responses of offspring. Indeed, animals experiencing non-nurturing maternal care show increased anxiety-like behaviour and enhanced stress responses in addition to epigenetic alterations (McGowan et al., 2011; Meaney and Szyf, 2005; Weaver et al., 2004). Maternal care-taking behaviour can be disrupted in alcoholic mothers (Christensen and Bilenberg, 2000; Jacobson, 1997). However, our results reveal no robust changes in 
maternal care behaviour due to binge alcohol consumption in our experimental conditions, suggesting that epigenetic changes observed in the brain of adult offspring mice seem to be directly related to the teratogenic effects of early alcohol exposure. Furthermore, there was no compromised nurturing effect on pup's body growth, suggesting that maternal care was appropriated in our experimental conditions.

Histone modification is one of the epigenetic mechanisms that can alter the molecular transcriptional machinery (Renthal and Nestler, 2008), and the covalent modifications to the tails of histones 3 and 4 ( $\mathrm{H} 3$ and $\mathrm{H} 4$, respectively) are the most commonly studied. Our results show that prenatal and postnatal (lactation) alcohol exposure induces an increase in the acetylation levels of lysines 5 and 12 of histone 4 (H4K5 and H4K12, respectively) and total K-ac in the PFC, while in the HPC only the acetylated levels of H4K5 were increased. Thus, indicating that developmental alcohol exposure induces region-specific epigenetic modifications in the brain. Furthermore, H4K5ac levels in the PFC correlated with the levels of maternal alcohol intake, suggesting a dose-dependent effect of alcohol on the acetylation of H4K5. Histone acetylation has been associated with an open chromatin structure; thus, our results would indicate an active state of gene transcription in the PFC of adult offspring mice due to binge-like alcohol exposure during prenatal and early postnatal periods. According with our results, increased levels of H4K5 and H4K12 acetylation have been reported after binge alcohol drinking in adolescent rodents (Montesinos et al., 2016; Pascual et al., 2009).

The enriched-histone acetylation levels observed in PLAE mice may be due to an increase in acetyl-CoA, the substrate needed for protein acetylation, as a result of alcohol metabolism (Chater-Diehl et al., 2017; Mandal et al., 2017), and also due to the modulation of the enzymes controlling such processes (HATs, HDACs) (Walker et al., 2015). Consequently, trying to elucidate the mechanism underlying such epigenetic changes, we measured the activity of HAT and HDAC enzymes. PLAE mice showed enhanced activity of HAT enzyme in the PFC, suggesting that long-term epigenetic alterations induced by early alcohol exposure in the PFC might be mediated by a dysregulation of the chromatin-modifying enzymes. Still, mechanisms involved in the augmented H4K5 acetylation in the HPC remain to be elucidated.

Other FASD preclinical studies focusing on epigenetics showed a global increase in H3K9 histone acetylation (Peng et al., 2015) and enhanced H3K14 acetylation in foetal 
hearts (Zhang et al., 2014), associated with an upregulated HAT activity without affecting HDAC activity. Similarly, induction of H3K14 acetylation at G9a exon 1 in the brain (Subbanna, et al., 2014a) and elevation of H4K8 acetylation at CB1R exon1 was observed after alcohol exposure at PD7 (Subbanna, et al., 2014b). However, it has been reported a global decrease in histone $\mathrm{H} 3$ and $\mathrm{H} 4$ acetylation in the cerebellum after perinatal alcohol vapour exposure, which has been proposed to account for the motor deficits associated with FASD (Guo et al., 2011). Furthermore, increased HDAC2 and HDAC4 expression has been reported in female offspring rats exposed to a liquid diet containing alcohol during gestation (Gangisetty et al., 2015). In another study, decreased histone activation marks ( $\mathrm{H} 3 \mathrm{~K} 4 \mathrm{me} 3$ and $\mathrm{H} 3 \mathrm{~K} 9 \mathrm{ac})$ and increased repressive marks (H3K9me2) were found in the rat hypothalamus due to foetal alcohol exposure (Bekdash et al., 2013). Nevertheless, in our study no changes in H3K9ac and H3K4me3 were found in the brain areas assessed. Such contrasting findings suggest that the alcohol-mediated epigenetic alterations are context-dependent (Mandal et al., 2017).

In the present study, we reported subtle alterations in spatial working memory and cognitive flexibility in PLAE offspring as assessed by the left-right discrimination task, in which different brain areas are involved, such as the PFC and the HPC (Deacon and Rawlins, 2006). The number of trials needed to reach criterion in the acquisition phase was higher in PLAE mice, suggesting impairments in the acquisition learning. However, no significant differences between groups were found during the reversal phase neither on the latency to reach the platform. Thus, mild cognitive deficits are here reported in PLAE offspring mice at adulthood. We have previously reported longlasting deficits in spatial working memory in PLAE mice, as assessed in the Y-maze, correlating with the levels of maternal alcohol intake (Cantacorps et al., 2017). Similarly, recent evidence indicates that heavy alcohol consumption during gestation induces adverse consequences in offspring cognition and behaviour (Murray et al., 2016; Zuccolo et al., 2013) that could be associated with persistent epigenetic changes and, particularly, in the modification of histones (Mandal et al., 2017).

Indeed, epigenetic regulatory mechanisms have been demonstrated to play a role in memory and learning processes (Nelson and Monteggia, 2011; Sen, 2015), such as acetylation and methylation of chromatin mediated by HAT and HDAC activities. Furthermore, H4K5ac and H4K12ac are key acetylation marks of memory-associated gene expression. Numerous studies have identified a correlation between this marks of 
histone acetylation with transcriptional activity involved in long-term memory formation and synaptic plasticity (Park et al., 2013; Peixoto and Abel, 2013; Peleg et al., 2010). In addition, promoting histone acetylation through HDAC inhibition improves memory formation (Penney and Tsai, 2014; Villain et al., 2016; Volmar and Wahlestedt, 2015). However, our results show significant elevated HAT activity, associated with increased histone acetylation, in the brain of PLAE mice, which present cognitive impairments. Therefore, we hypothesize that other mechanisms might be involved in these memory and learning alterations induced by developmental binge alcohol exposure, such as disrupted functional brain connectivity as previously reported (Cantacorps et al., 2018).

Furthermore, we have previously showed that maternal alcohol drinking promotes proinflammatory and neurodegenerative processes in the offspring's brain, and some of these processes are maintained into adulthood (Cantacorps et al., 2017), suggesting that alcohol exposure during critical stages of the developing brain induces long-lasting neuroimmune alterations associated with epigenetic changes together with alcoholrelated impairments in learning, memory and behavioural dysfunctions. It is important to emphasise that the molecular regulation of neuroinflammation is strongly influenced by epigenetic mechanisms (Garden, 2013). Changes in histone acetylation, HDAC activity and neuroinflammation, in addition to upregulated TLR4 response, have been reported in the cortex of adult mice treated with alcohol (Pascual et al., 2011). Likewise, a recent study also demonstrated that TLR4 inflammatory response participates in the neurodevelopmental alterations and the long-lasting behavioural dysfunctions associated with FASD (Pascual et al., 2017). Moreover, alterations in myelin proteins have been reported in our model, suggesting that neuronal transmission is impaired and thus, functional networks are altered, which may be contributing to the behavioural defects (Cantacorps et al., 2017, 2018).

In summary, our results indicate that binge-like alcohol exposure during gestation and lactation periods induces long-lasting epigenetic alterations in the brain. These changes might be related with the modulation of neuroinflammatory responses and abnormal neuroplasticity alterations during brain development, presumably contributing to the appearance of cognitive and behavioural dysfunctions. 


\section{Conclusions}

The current study reports persistent epigenetic modifications in the brain and spatial learning dysfunctions in mice after pre- and postnatal alcohol exposure. Specifically, maternal binge-like alcohol consumption during gestation and lactation periods induced an increase in the acetylation of specific histone marks in the PFC and HPC of PLAE mice, as well as, long-lasting mild cognitive deficits.

Further studies using enzyme-specific and candidate gene approaches would be essential for understanding the underlying mechanism to the neurobehavioural alterations associated with pre- and postnatal binge alcohol exposure. More knowledge about the contribution of the epigenetic regulation to specific gene expression changes altered by alcohol exposure may provide us with the tools for developing new biomarkers and treatments to prevent alcohol-mediated teratogenic effects such as those observed in FASD.

\section{Acknowledgements}

We thank Gerald Fannon for English proofreading and manuscript editing. This study was supported by grants from the European Union's Horizon 2020 research and innovation program 2014-2020 under Grant Agreement No 634143, Spanish Ministry of Economy, Innovation and Competiveness (SAF2015-69187-R-FEDER; SAF201675347-R) and Spanish Ministry of Health (Retic-ISCIII-RD/12/0028/007 and RD/16/0017/0010-FEDER and Plan Nacional sobre Drogas \#2014/020, \#2015/010 and \#2018/007). The Department of Experimental and Health Sciences (UPF) is a "Unidad de Excelencia María de Maeztu" funded by the MINECO (Ref. MDM-2014-0370). 


\section{Figure legends}

Figure 1. Diagram of experimental procedure.

Figure 2. Maternal binge-like alcohol intake. Maternal alcohol intake $(\mathrm{gEtOH} / \mathrm{kg})$ during weekly binge sessions (4h access) of DID procedure throughout gestation and lactation periods (One-way ANOVA with repeated measures). Data are expressed as [mean $\pm \mathrm{SEM}](\mathrm{n}=14)$.

Figure 3. Effects of maternal alcohol consumption on maternal care behaviour. Archedback posture (A), blanket nursing posture (B), passive nursing posture (C) and off-nest behaviour (D) of dams exposed to DID test during prenatal and lactation periods. * $\mathrm{p}<0.05$ (Oneway ANOVA with repeated measures and Bonferroni post-hoc test). Data are expressed as $[$ mean $\pm \mathrm{SEM}](\mathrm{n}=6$ per group).

Figure 4. Left-right discrimination learning. Latency to reach the platform during the last five trials to criterion during acquisition and reversal (A). Number of trials needed to reach criterion in acquisition and reversal phases (B). ${ }^{*} \mathrm{p}<0.05$ (Student's t-test). Values are presented as $[$ mean \pm SEM] ( $n=10$ per group).

Figure 5. Long-term epigenetic changes in histone acetylation and methylation due to early alcohol exposure. Western blot analyses show the levels of histone acetylation for $\mathrm{H} 3 \mathrm{~K} 9$, H4K5, H4K12, K-ac, and histone methylation for H3K4me3 in the PFC (A) and HPC (B) of control and PLAE mice. Lamin $\mathrm{A} / \mathrm{C}$ was used as a loading control. $* \mathrm{p}<0.05, * * \mathrm{p}<0.01$, $* * * \mathrm{p}<0.001$ (Student's t-test). Bars are presented as [mean $\pm \mathrm{SEM}]$ ( $\mathrm{n}=3-5$ per group).

Figure 6. Effects of early alcohol exposure on the enzyme activity of epigenetic modifiers. HAT (A) and HDAC (B) activity was measured in the PFC and HPC of control and PLAE mice. ${ }^{*} \mathrm{p}<0.05$ (Student's t-test). Values are presented as [mean $\left.\pm \mathrm{SEM}\right]$ ( $\mathrm{n}=3-5$ per group).

\section{Table notes}

Table 1. Primary antibodies.

Table 2. Two-way ANOVA calculated for maternal care behaviour of dams exposed to the DID test during the prenatal period and postnatal periods.

Table 3. Litter size and average pup weight. Values are [mean \pm SEM].

Table 4. Unpaired Student's t-test for histone modification measurements in the PFC and the HPC of PAE and PLAE mice compared with control group. 


\section{References}

Alfonso-Loeches S and Guerri C (2011) Molecular and behavioral aspects of the actions of alcohol on the adult and developing brain. Critical reviews in clinical laboratory sciences 48(1): 19-47. DOI: 10.3109/10408363.2011.580567.

Alfonso-Loeches S, Pascual-Lucas M, Blanco AM, et al. (2010) Pivotal role of TLR4 receptors in alcohol-induced neuroinflammation and brain damage. The Journal of neuroscience : the official journal of the Society for Neuroscience 30(24): 8285-95. DOI: 10.1523/JNEUROSCI.0976-10.2010.

Banik A, Kandilya D, Ramya S, et al. (2017) Maternal Factors that Induce Epigenetic Changes Contribute to Neurological Disorders in Offspring. Genes 8(6). DOI: 10.3390/genes8060150.

Barron S, Hawkey A, Fields L, et al. (2016) Animal Models for Medication Development and Application to Treat Fetal Alcohol Effects. International review of neurobiology 126: 423-40. DOI: 10.1016/bs.irn.2016.02.002.

Basavarajappa B and Subbanna S (2016) Epigenetic Mechanisms in Developmental AlcoholInduced Neurobehavioral Deficits. Brain Sciences 6(2): 12. DOI: 10.3390/brainsci6020012.

Bekdash RA, Zhang C and Sarkar DK (2013) Gestational choline supplementation normalized fetal alcohol-induced alterations in histone modifications, DNA methylation, and proopiomelanocortin (POMC) gene expression in $\beta$-endorphin-producing POMC neurons of the hypothalamus. Alcoholism, clinical and experimental research 37(7): 1133-42. DOI: 10.1111/acer.12082.

Brady ML, Allan AM and Caldwell KK (2012) A limited access mouse model of prenatal alcohol exposure that produces long-lasting deficits in hippocampal-dependent learning and memory. Alcoholism, clinical and experimental research 36(3): 457-66. DOI: 10.1111/j.1530-0277.2011.01644.x.

Cantacorps L, Alfonso-Loeches S, Moscoso-Castro M, et al. (2017) Maternal alcohol binge drinking induces persistent neuroinflammation associated with myelin damage and behavioural dysfunctions in offspring mice. Neuropharmacology 123: 368-384. DOI: 10.1016/j.neuropharm.2017.05.034.

Cantacorps L, González-Pardo H, Arias JL, et al. (2018) Altered brain functional connectivity and behaviour in a mouse model of maternal alcohol binge-drinking. Progress in neuro- 
psychopharmacology \& biological psychiatry 84(Pt A). Elsevier Inc: 237-249. DOI: 10.1016/j.pnpbp.2018.03.006.

Chater-Diehl EJ, Laufer BI and Singh SM (2017) Changes to histone modifications following prenatal alcohol exposure: An emerging picture. Alcohol (Fayetteville, N.Y.) 60. Elsevier Ltd: 41-52. DOI: 10.1016/j.alcohol.2017.01.005.

Christensen HB and Bilenberg N (2000) Behavioural and emotional problems in children of alcoholic mothers and fathers. European child \& adolescent psychiatry 9(3): 219-226. DOI: $10.1007 / \mathrm{s} 007870070046$.

Deacon RMJ and Rawlins JNP (2006) T-maze alternation in the rodent. Nature Protocols 1(1). Nature Publishing Group: 7-12. DOI: 10.1038/nprot.2006.2.

Dörrie N, Föcker M, Freunscht I, et al. (2014) Fetal alcohol spectrum disorders. European child \& adolescent psychiatry 23(10): 863-75. DOI: 10.1007/s00787-014-0571-6.

Drew PD and Kane CJM (2014) Fetal alcohol spectrum disorders and neuroimmune changes. International review of neurobiology 118: 41-80. DOI: 10.1016/B978-0-12-8012840.00003-8.

Filali M and Lalonde R (2009) Age-related cognitive decline and nesting behavior in an APPswe/PS1 bigenic model of Alzheimer's disease. Brain research 1292. Elsevier B.V.: 93-9. DOI: 10.1016/j.brainres.2009.07.066.

Fodor A, Klausz B, Pintér O, et al. (2012) Maternal neglect with reduced depressive-like behavior and blunted c-fos activation in Brattleboro mothers, the role of central vasopressin. Hormones and behavior 62(4): 539-51. DOI: 10.1016/j.yhbeh.2012.09.003.

Gangisetty O, Wynne O, Jabbar S, et al. (2015) Fetal Alcohol Exposure Reduces Dopamine Receptor D2 and Increases Pituitary Weight and Prolactin Production via Epigenetic Mechanisms. PloS one 10(10): e0140699. DOI: 10.1371/journal.pone.0140699.

Garden GA (2013) Epigenetics and the Modulation of Neuroinflammation. Neurotherapeutics 10(4): 782-788. DOI: 10.1007/s13311-013-0207-4.

Gracia-Rubio I, Moscoso-Castro M, Pozo OJ, et al. (2016) Maternal separation induces neuroinflammation and long-lasting emotional alterations in mice. Progress in neuropsychopharmacology \& biological psychiatry 65. Elsevier Inc.: 104-17. DOI: 10.1016/j.pnpbp.2015.09.003.

Guerri C, Bazinet A and Riley EP (2009) Foetal Alcohol Spectrum Disorders and alterations in 
brain and behaviour. Alcohol and alcoholism (Oxford, Oxfordshire) 44(2): 108-14. DOI: 10.1093/alcalc/agn105.

Guo W, Crossey EL, Zhang L, et al. (2011) Alcohol exposure decreases CREB binding protein expression and histone acetylation in the developing cerebellum. PLOS ONE 6(5). DOI: 10.1371/journal.pone.0019351.

Haycock PC (2009) Fetal alcohol spectrum disorders: the epigenetic perspective. Biology of reproduction 81(4): 607-17. DOI: 10.1095/biolreprod.108.074690.

Hoyme HE, Kalberg WO, Elliott AJ, et al. (2016) Updated Clinical Guidelines for Diagnosing Fetal Alcohol Spectrum Disorders. Pediatrics 138(2): e20154256-e20154256. DOI: 10.1542/peds.2015-4256.

Jacobson SW (1997) Assessing the impact of maternal drinking during and after pregnancy. Alcohol health and research world 21(3): 199-203.

Keverne EB, Pfaff DW and Tabansky I (2015) Epigenetic changes in the developing brain: Effects on behavior. Proceedings of the National Academy of Sciences 112(22): 67896795. DOI: 10.1073/pnas.1501482112.

Kleiber ML, Diehl EJ, Laufer BI, et al. (2014) Long-term genomic and epigenomic dysregulation as a consequence of prenatal alcohol exposure: A model for fetal alcohol spectrum disorders. Frontiers in Genetics 5: 161. DOI: 10.3389/fgene.2014.00161.

Krishnan HR, Sakharkar AJ, Teppen TL, et al. (2014) The epigenetic landscape of alcoholism. International review of neurobiology 115(312): 75-116. DOI: 10.1016/B978-0-12801311-3.00003-2.

Kundakovic M and Champagne FA (2015) Early-life experience, Epigenetics, and the developing brain. Neuropsychopharmacology 40(1). Nature Publishing Group: 141-153. DOI: 10.1038/npp.2014.140.

Lange S, Probst C, Gmel G, et al. (2017) Global Prevalence of Fetal Alcohol Spectrum Disorder Among Children and Youth: A Systematic Review and Meta-analysis. JAMA pediatrics 171(10): 948-956. DOI: 10.1001/jamapediatrics.2017.1919.

Lara AH and Wallis JD (2015) The Role of Prefrontal Cortex in Working Memory: A Mini Review. Frontiers in Systems Neuroscience 9(8): 372-376. DOI:

10.3389/fnsys.2015.00173.

Liu Y, Balaraman Y, Wang G, et al. (2009) Alcohol exposure alters DNA methylation profiles 
in mouse embryos at early neurulation. Epigenetics : official journal of the DNA

Methylation Society 4(7): 500-11. DOI: 10.1111/j.1600-6143.2008.02497.x.Plasma.

Liyanage VRB, Curtis K, Zachariah RM, et al. (2017) Overview of the Genetic Basis and Epigenetic Mechanisms that Contribute to FASD Pathobiology. Current topics in medicinal chemistry 17(7): 808-828. DOI: 10.2174/1568026616666160414124816.

Lussier AA, Weinberg J and Kobor MS (2017) Epigenetics studies of fetal alcohol spectrum disorder: where are we now? Epigenomics 9(3): 291-311. DOI: 10.2217/epi-2016-0163.

Mahnke AH, Miranda RC and Homanics GE (2017) Epigenetic mediators and consequences of excessive alcohol consumption. Alcohol (Fayetteville, N.Y.) 60(1): 1-6. DOI: 10.1016/j.alcohol.2017.02.357.

Mandal C, Halder D, Jung KH, et al. (2017) In utero alcohol exposure and the alteration of histone marks in the developing fetus: An epigenetic phenomenon of maternal drinking. International Journal of Biological Sciences 13(9): 1100-1108. DOI: 10.7150/ijbs.21047.

Mantha K, Laufer BI and Singh SM (2014) Molecular changes during neurodevelopment following second-trimester binge ethanol exposure in a mouse model of fetal alcohol spectrum disorder: From immediate effects to long-term adaptation. Developmental Neuroscience 36(1): 29-43. DOI: 10.1159/000357496.

Marjonen H, Sierra A, Nyman A, et al. (2015) Early maternal alcohol consumption alters hippocampal DNA methylation, gene expression and volume in a mouse model. PLoS ONE 10(5): 1-20. DOI: 10.1371/journal.pone.0124931.

May PA, Chambers CD, Kalberg WO, et al. (2018) Prevalence of fetal alcohol spectrum disorders in 4 US communities. JAMA - Journal of the American Medical Association 319(5): 474-482. DOI: 10.1001/jama.2017.21896.

McGowan PO, Suderman M, Sasaki A, et al. (2011) Broad epigenetic signature of maternal care in the brain of adult rats. PloS one 6(2): e14739. DOI: 10.1371/journal.pone.0014739.

Meaney MJ and Szyf M (2005) Maternal care as a model for experience-dependent chromatin plasticity? Trends in neurosciences 28(9): 456-63. DOI: 10.1016/j.tins.2005.07.006.

Montesinos J, Pascual M, Rodríguez-Arias M, et al. (2016) Involvement of TLR4 in the longterm epigenetic changes, rewarding and anxiety effects induced by intermittent ethanol treatment in adolescence. Brain, Behavior, and Immunity 53. Elsevier Inc.: 159-71. DOI: 10.1016/j.bbi.2015.12.006. 
Murray J, Burgess S, Zuccolo L, et al. (2016) Moderate alcohol drinking in pregnancy increases risk for children's persistent conduct problems: Causal effects in a Mendelian randomisation study. Journal of Child Psychology and Psychiatry and Allied Disciplines 57(5): 575-584. DOI: 10.1111/jcpp.12486.

Nelson ED and Monteggia LM (2011) Epigenetics in the mature mammalian brain: Effects on behavior and synaptic transmission. Neurobiology of Learning and Memory 96(1). Elsevier Inc.: 53-60. DOI: 10.1016/j.nlm.2011.02.015.

Park C, Rehrauer H and Mansuy IM (2013) Genome-wide analysis of H4K5 acetylation associated with fear memory in mice. BMC Genomics 14(1): 539. DOI: 10.1186/14712164-14-539.

Pascual M, Boix J, Felipo V, et al. (2009) Repeated alcohol administration during adolescence causes changes in the mesolimbic dopaminergic and glutamatergic systems and promotes alcohol intake in the adult rat. Journal of Neurochemistry 108(4): 920-931. DOI: 10.1111/j.1471-4159.2008.05835.x.

Pascual M, Baliño P, Alfonso-Loeches S, et al. (2011) Impact of TLR4 on behavioral and cognitive dysfunctions associated with alcohol-induced neuroinflammatory damage. Brain, Behavior, and Immunity 25(SUPPL. 1): 80-91. DOI: 10.1016/j.bbi.2011.02.012.

Pascual M, Montesinos J, Montagud-Romero S, et al. (2017) TLR4 response mediates ethanolinduced neurodevelopment alterations in a model of fetal alcohol spectrum disorders. Journal of Neuroinflammation 14(1): 145. DOI: 10.1186/s12974-017-0918-2.

Peixoto L and Abel T (2013) The role of histone acetylation in memory formation and cognitive impairments. Neuropsychopharmacology 38(1). Nature Publishing Group: 62-76. DOI: 10.1038/npp.2012.86.

Peleg S, Sananbenesi F, Zovoilis A, et al. (2010) Altered Histone Acetylation Is Associated with Age-Dependent Memory Impairment in Mice. Science 328(5979): 753-756. DOI: 10.1126/science.1186088.

Peng C, Zhang W, Zhao W, et al. (2015) Alcohol-induced histone H3K9 hyperacetylation and cardiac hypertrophy are reversed by a histone acetylases inhibitor anacardic acid in developing murine hearts. Biochimie 113. Elsevier Ltd: 1-9. DOI: 10.1016/j.biochi.2015.03.012.

Penney J and Tsai L-H (2014) Histone deacetylases in memory and cognition. Science Signaling 7(355): re12-re12. DOI: 10.1126/scisignal.aaa0069. 
Petanjek Z and Kostovic I (2012) Epigenetic regulation of fetal brain development and neurocognitive outcome. Proceedings of the National Academy of Sciences 109(28): 11062-11063. DOI: 10.1073/pnas.1208085109.

Ponomarev I (2013) Epigenetic control of gene expression in the alcoholic brain. Alcohol research: current reviews 35(1): 69-76.

Popova S, Lange S, Probst C, et al. (2017) Prevalence of alcohol consumption during pregnancy and Fetal Alcohol Spectrum Disorders among the general and Aboriginal populations in Canada and the United States. European Journal of Medical Genetics 60(1). Elsevier Masson SAS: 32-48. DOI: 10.1016/j.ejmg.2016.09.010.

Ramsay M (2010) Genetic and epigenetic insights into fetal alcohol spectrum disorders. Genome medicine 2(4): 27. DOI: 10.1186/gm148.

Renthal W and Nestler EJ (2008) Epigenetic mechanisms in drug addiction. Trends in Molecular Medicine 14(8): 341-350. DOI: 10.1016/j.molmed.2008.06.004.

Resendiz M, Chen Y, Oztürk NC, et al. (2013) Epigenetic medicine and fetal alcohol spectrum disorders. Epigenomics 5(1): 73-86. DOI: 10.2217/epi.12.80.

Resendiz M, Mason S, Lo CL, et al. (2014) Epigenetic regulation of the neural transcriptome and alcohol interference during development. Frontiers in Genetics 5: 285. DOI: 10.3389/fgene.2014.00285.

Rhodes JS, Best K, Belknap JK, et al. (2005) Evaluation of a simple model of ethanol drinking to intoxication in C57BL/6J mice. Physiology \& behavior 84(1): 53-63. DOI: 10.1016/j.physbeh.2004.10.007.

Rubin RD, Watson PD, Duff MC, et al. (2014) The role of the hippocampus in flexible cognition and social behavior. Frontiers in Human Neuroscience 8(September): 1-15. DOI: $10.3389 /$ fnhum.2014.00742.

Sadrian B, Lopez-Guzman M, Wilson D a, et al. (2014) Distinct neurobehavioral dysfunction based on the timing of developmental binge-like alcohol exposure. Neuroscience 280. IBRO: 204-19. DOI: 10.1016/j.neuroscience.2014.09.008.

Sen N (2015) Epigenetic Regulation of Memory by Acetylation and Methylation of Chromatin: Implications in Neurological Disorders, Aging, and Addiction. NeuroMolecular Medicine 17(2). Springer US: 97-110. DOI: 10.1007/s12017-014-8306-x.

Shukla SD, Velazquez J, French SW, et al. (2008) Emerging role of epigenetics in the actions of 
alcohol. Alcoholism: Clinical and Experimental Research 32(9): 1525-1534. DOI: 10.1111/j.1530-0277.2008.00729.x.

Sigurdsson T and Duvarci S (2016) Hippocampal-Prefrontal Interactions in Cognition, Behavior and Psychiatric Disease. Frontiers in systems neuroscience 9(January): 190. DOI: 10.3389/fnsys.2015.00190.

Subbanna S, Nagre NN, Shivakumar M, et al. (2014a) Ethanol induced acetylation of histone at G9a exon1 and G9a-mediated histone $\mathrm{H} 3$ dimethylation leads to neurodegeneration in neonatal mice. Neuroscience 258. IBRO: 422-32. DOI:

10.1016/j.neuroscience.2013.11.043.

Subbanna S, Nagre NN, Umapathy NS, et al. (2014b) Ethanol exposure induces neonatal neurodegeneration by enhancing CB1R Exon1 histone H4K8 acetylation and up-regulating CB1R function causing neurobehavioral abnormalities in adult mice. The international journal of neuropsychopharmacology 18(5): pyu028. DOI: 10.1093/ijnp/pyu028.

Sweatt JD (2010) Epigenetics and Cognitive Aging. Science 328(5979): 701-702. DOI: 10.1126/science.1189968.

Ungerer M, Knezovich J and Ramsay M (2013) In utero alcohol exposure, epigenetic changes, and their consequences. Alcohol research : current reviews 35(1): 37-46.

Villain H, Florian C and Roullet P (2016) HDAC inhibition promotes both initial consolidation and reconsolidation of spatial memory in mice. Scientific reports 6(May). Nature Publishing Group: 27015. DOI: 10.1038/srep27015.

Volmar CH and Wahlestedt C (2015) Histone deacetylases (HDACs) and brain function. Neuroepigenetics 1. The Authors: 20-27. DOI: 10.1016/j.nepig.2014.10.002.

Walker DM, Cates HM, Heller EA, et al. (2015) Regulation of chromatin states by drugs of abuse. Current Opinion in Neurobiology 30(Figure 2). Elsevier Ltd: 112-121. DOI: 10.1016/j.conb.2014.11.002.

Weaver ICG, Cervoni N, Champagne F a, et al. (2004) Epigenetic programming by maternal behavior. Nature Neuroscience 7(8): 847-854. DOI: 10.1038/nn1276.

Zakhari S (2013) Alcohol metabolism and epigenetics changes. Alcohol research : current reviews 35(1): 6-16.

Zhang W, Peng C, Zheng M, et al. (2014) Prenatal alcohol exposure causes the over-expression of DHAND and EHAND by increasing histone H3K14 acetylation in C57 BL/6 mice. 
Toxicology letters 228(3). Elsevier Ireland Ltd: 140-6. DOI: 10.1016/j.toxlet.2014.05.011.

Zuccolo L, Lewis SJ, Smith GD, et al. (2013) Prenatal alcohol exposure and offspring cognition and school performance. A mendelian randomization natural experiment. International Journal of Epidemiology 42(5): 1358-1370. DOI: 10.1093/ije/dyt172. 
Fig. 1 Diagram of experimental procedure
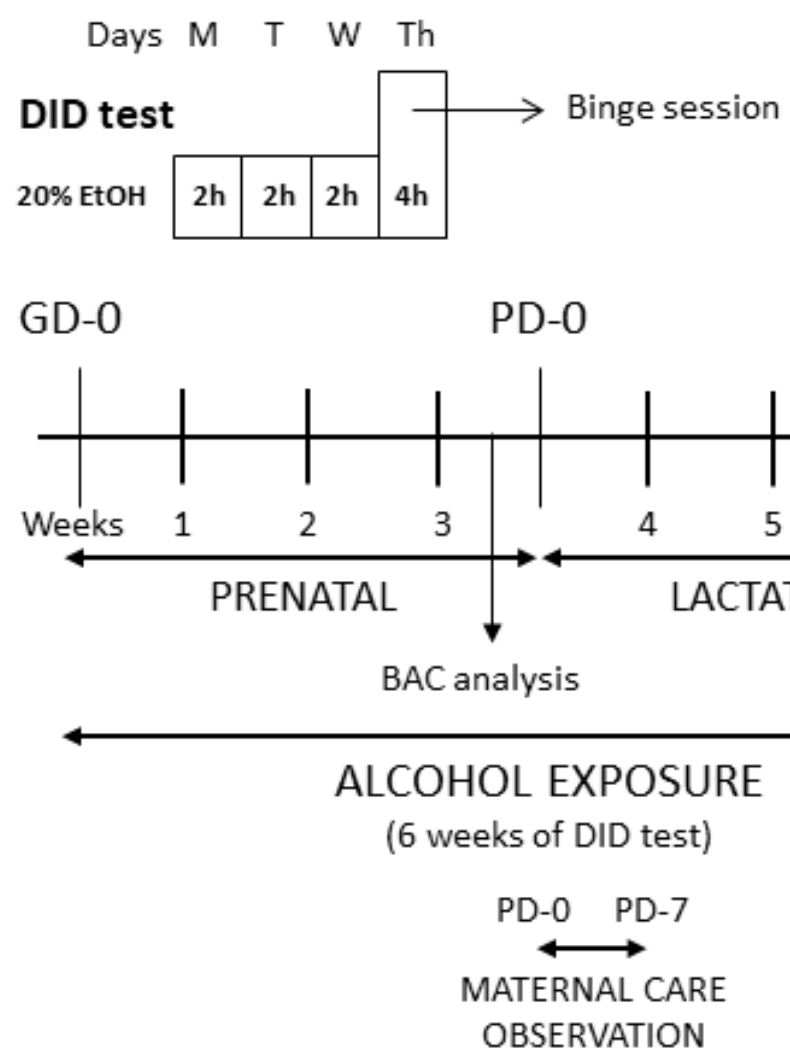

PD-21

PD-60

PD-70

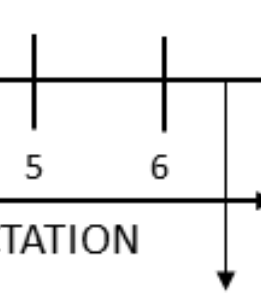

BAC analysis

T-MAZE

BRAIN TISSUE COLLECTION

OBSERVATION 
Fig. 2 Maternal binge-like alcohol consumption

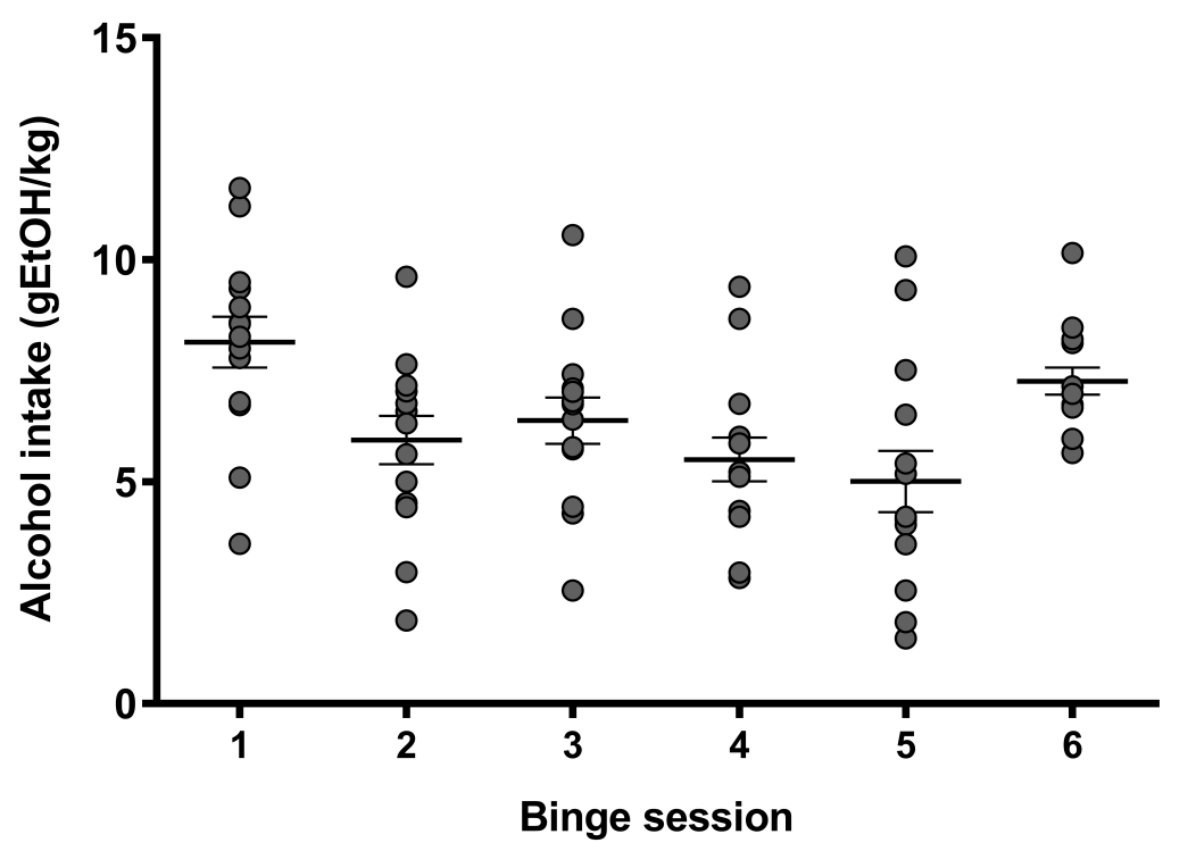


Fig. 3 Maternal care behaviour

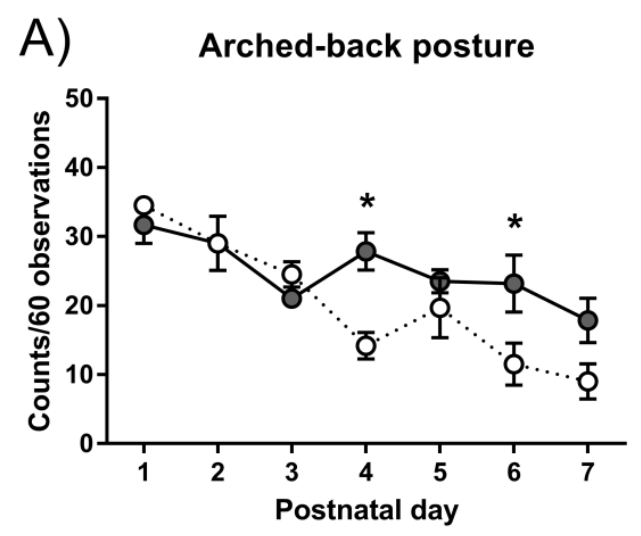

B) Blanket nursing posture

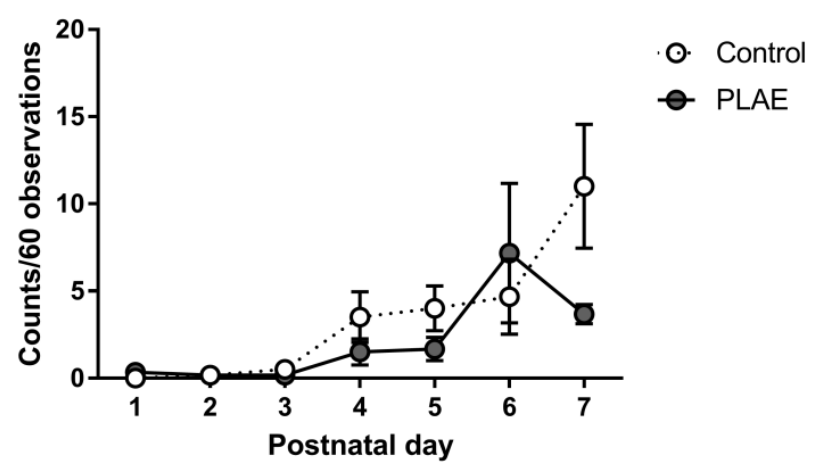

C) Passive nursing posture
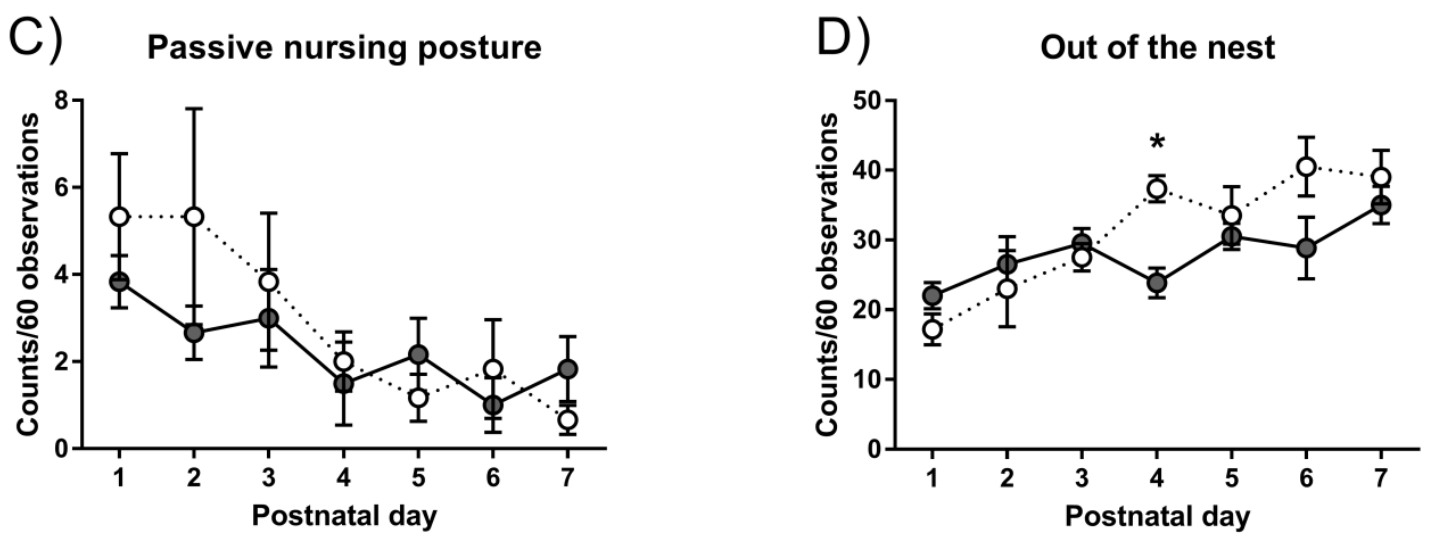
Fig. 4 Reversal learning T-maze

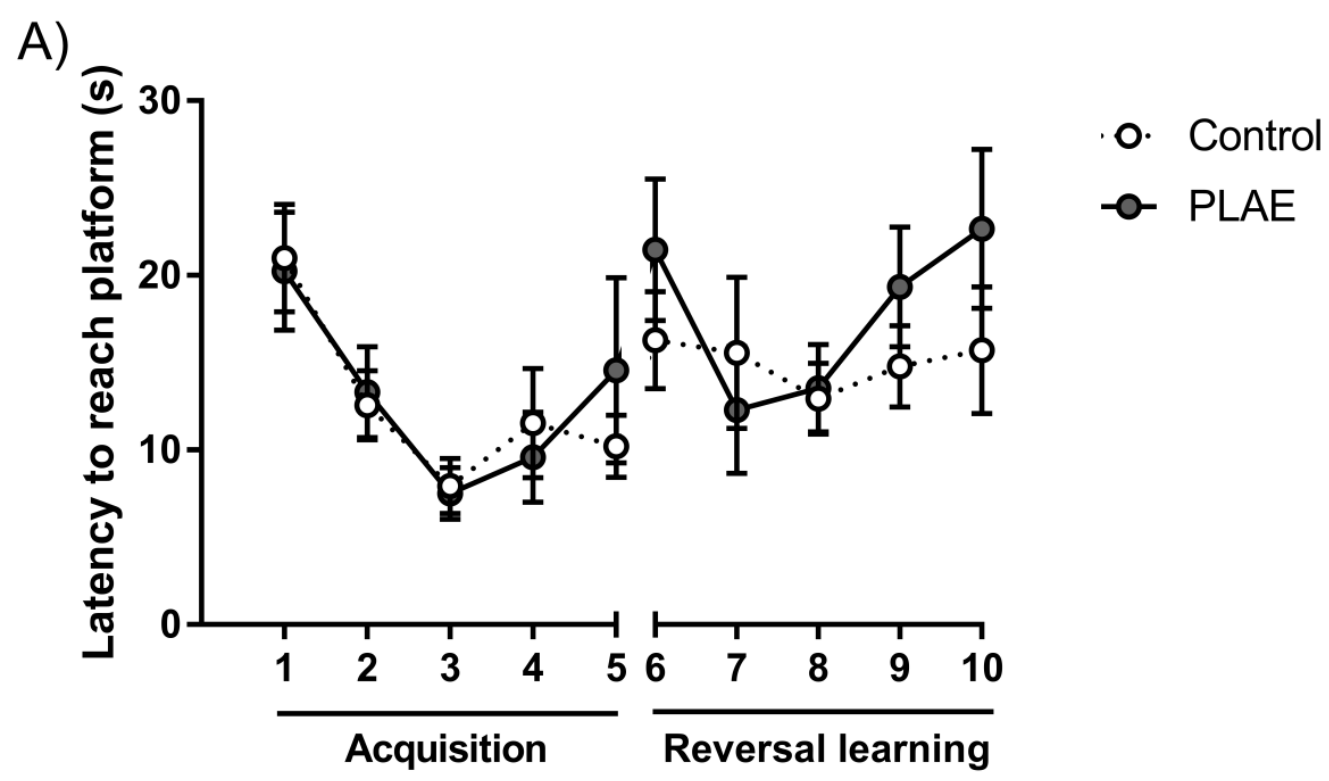

B)

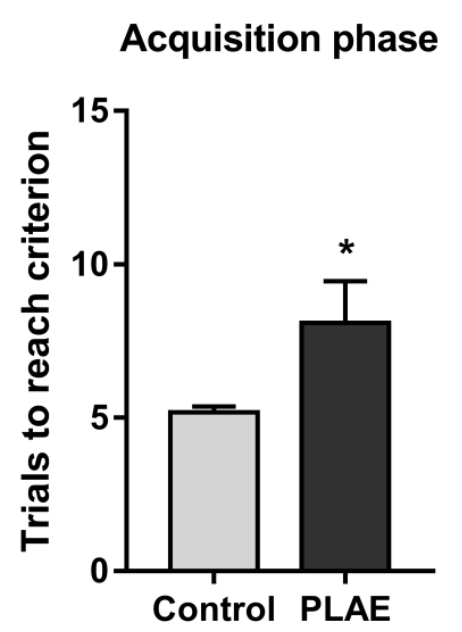

Reversal learning phase

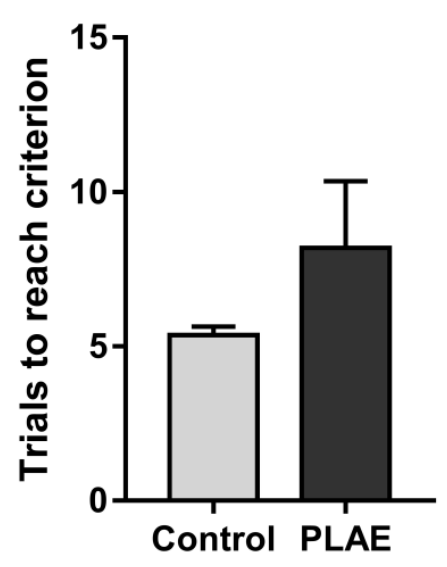


Fig. 5 Histone modifications
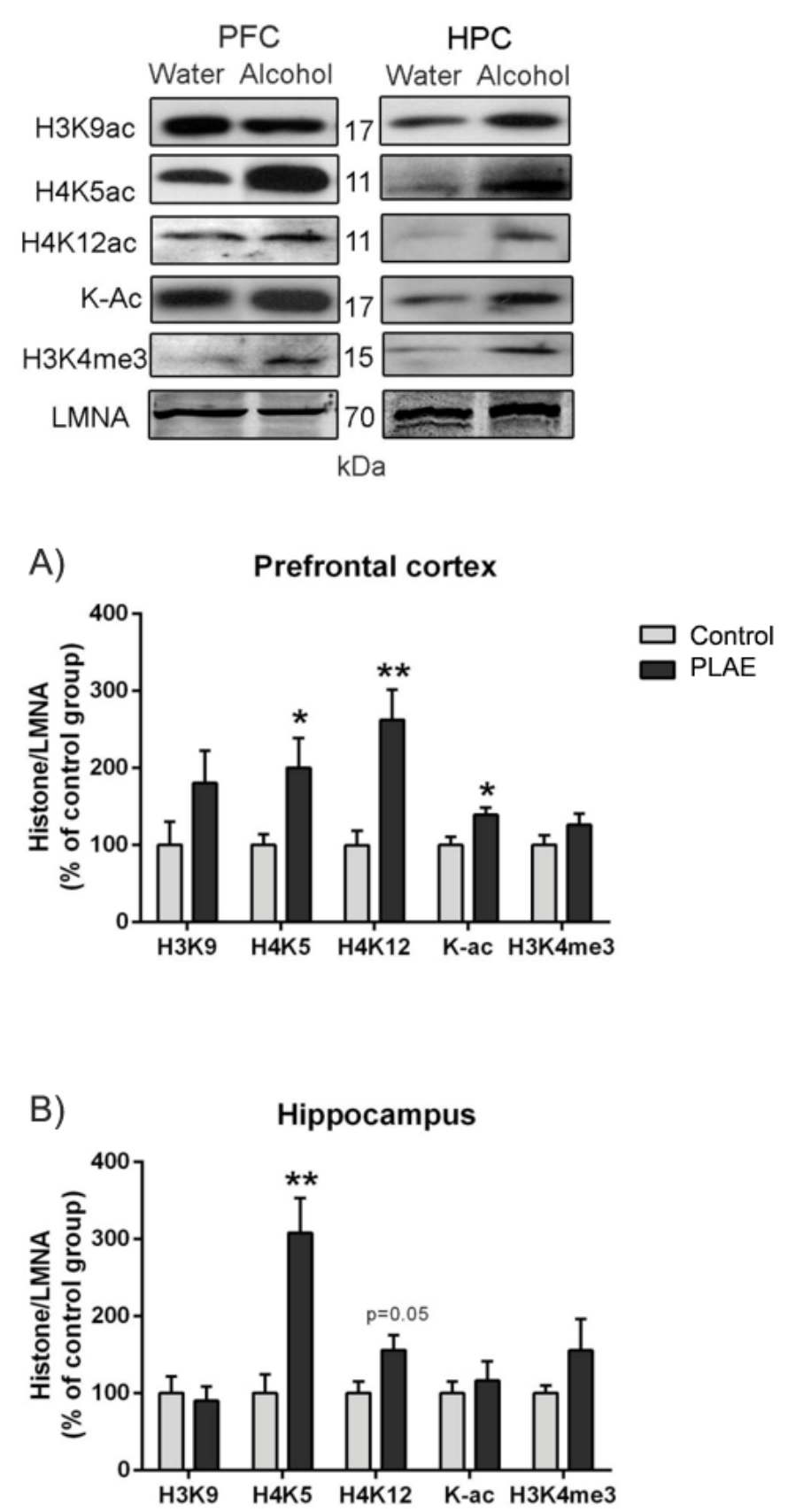
Fig. 6 HAT and HDAC activities
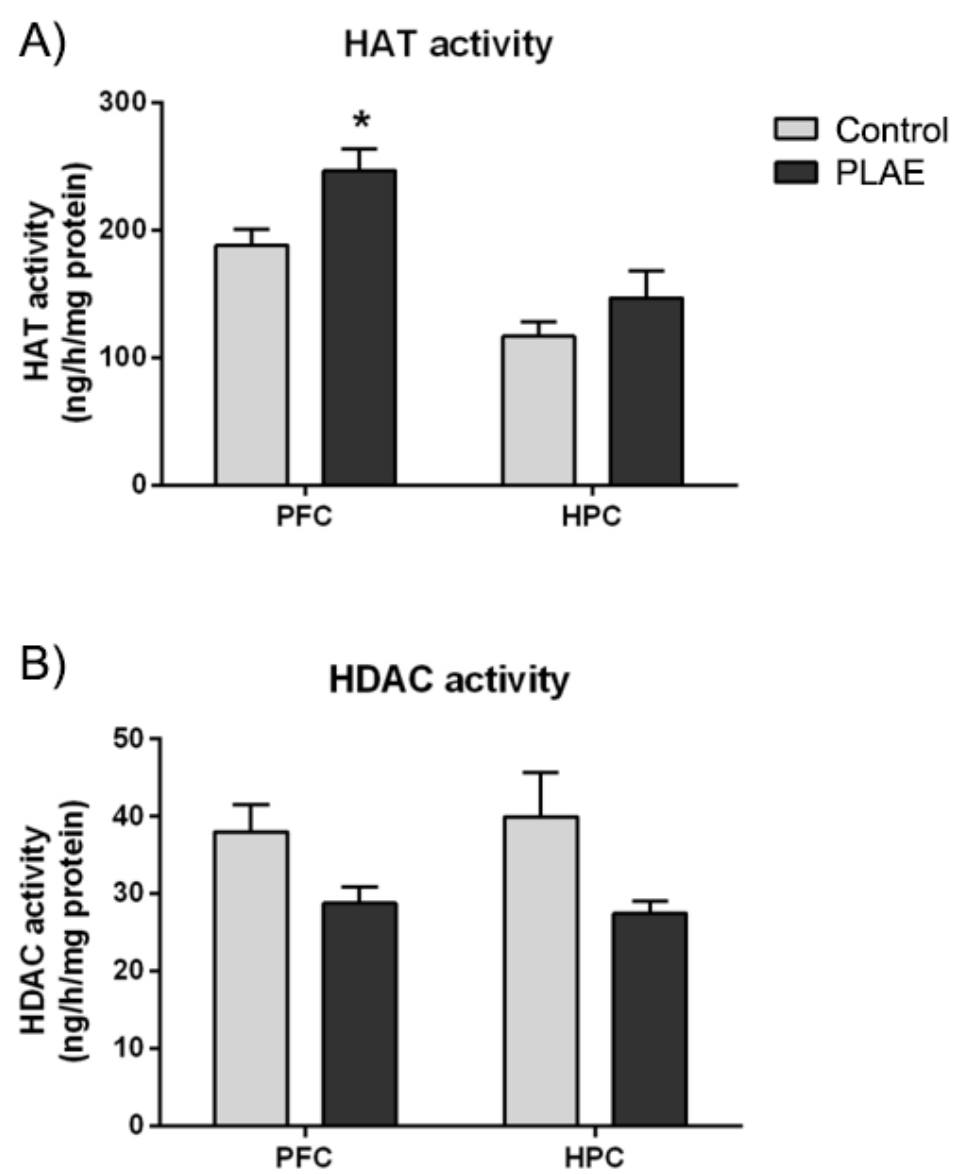